\title{
Type IIP supernova 2008in: the explosion of a normal red supergiant
}

\author{
V. P. Utrobin ${ }^{1,2}$ and N. N. Chugai ${ }^{3}$ \\ ${ }^{1}$ Max-Planck-Institut für Astrophysik, Karl-Schwarzschild-Str. 1, 85748 Garching, Germany \\ e-mail: utrobin@itep.ru \\ ${ }^{2}$ Institute of Theoretical and Experimental Physics, B. Cheremushkinskaya St. 25, 117218 Moscow, Russia \\ ${ }^{3}$ Institute of Astronomy of Russian Academy of Sciences, Pyatnitskaya St. 48, 119017 Moscow, Russia
}

Received 10 April 2013 / Accepted 5 June 2013

\begin{abstract}
Context. The explosion energy and the ejecta mass of a type IIP supernova make up the basis for the theory of explosion mechanism. So far, these parameters have only been determined for seven events.

Aims. Type IIP supernova 2008in is another well-observed event for which a detailed hydrodynamic modeling can be used to derive the supernova parameters.

Methods. Hydrodynamic modeling was employed to describe the bolometric light curve and the expansion velocities at the photosphere level. A time-dependent model for hydrogen ionization and excitation was applied to model the $\mathrm{H} \alpha$ and $\mathrm{H} \beta$ line profiles.

Results. We found an ejecta mass of $13.6 \pm 1.9 M_{\odot}$, an explosion energy of $(5.05 \pm 3.4) \times 10^{50} \mathrm{erg}$, a presupernova radius of $570 \pm 100 R_{\odot}$, and a radioactive ${ }^{56} \mathrm{Ni}$ mass of $0.015 \pm 0.005 M_{\odot}$. The estimated progenitor mass is $15.5 \pm 2.2 M_{\odot}$. We uncovered a problem of the $\mathrm{H} \alpha$ and $\mathrm{H} \beta$ description at the early phase, which cannot be resolved within a spherically symmetric model.

Conclusions. The presupernova of SN 2008in was a normal red supergiant with the minimum mass of the progenitor among eight type IIP supernovae explored by means of the hydrodynamic modeling. The problem of the absence of type IIP supernovae with the progenitor masses $<15 M_{\odot}$ in this sample remains open.
\end{abstract}

Key words. supernovae: individual: 2008in - supernovae: general

\section{Introduction}

Type IIP supernovae (SNe IIP) comprise roughly half of corecollapse SNe related to massive stars (Smith et al. 2011). The current theory predicts that SNe IIP originate from 9-25 $M_{\odot}$ stars (Heger et al. 2003), although the issue is still unsettled regarding the precise values of the lower and upper boundaries. It is well known that the luminosities of SNe IIP are distributed in a broad range, which is reflected in the categories of luminous, normal, subluminous, and faint events. What does determine the difference remains unclear, although there is a hint that the SN luminosity may be controlled by a progenitor mass, with the higher luminosity events coming out from the higher stellar masses (see Utrobin \& Chugai 2011, and references therein).

An understanding of SNe IIP phenomenon requires welldetermined SN parameters. However, only the radioactive ${ }^{56} \mathrm{Ni}$ mass can be directly estimated from the observed luminosity at the radioactive tail. A detailed hydrodynamic modeling is needed to reliably recover the parameter values of the ejecta mass, the explosion energy, and the pre-SN radius. In turn, the hydrodynamic modeling becomes an effective diagnostic exclusively in the case of the well-observed SNe IIP, which suggests a complete light curve from the rising part to the radioactive tail and a detailed spectral coverage. Unfortunately, only a handful of SN IIP events met these requirements which explained a scarcity of SNe IIP with the detailed hydrodynamic modeling (see Utrobin \& Chugai 2011).

Given this fact, we focus on SN 2008in, the recent SN IIP exploded in nearby galaxy M61 (NGC 4303) and well observed photometrically from the rising part to the radioactive tail (Roy et al. 2011). This is the second case, after SN 2005cs, of well-studied observationally subluminous SN IIP. Using scaling relations between the observables and the SN parameters, Roy et al. (2011) find the ejecta mass of $\sim 16.7 M_{\odot}$, the explosion energy of $\sim 5.4 \times 10^{50} \mathrm{erg}$, and the pre-SN radius of $\sim 126 R_{\odot}$ and conclude that SN 2008in is the result of a low-energy explosion of a relatively compact star. We would like to emphasize that the estimates based on the analytical relations between the observables (the plateau duration, the luminosity, and the velocity at the photosphere level in a certain epoch) and the SN parameters (the explosion energy, the ejecta mass, and the pre-SN radius) should be considered as approximate; a detailed hydrodynamic modeling of the light curve and the velocity evolution is needed to infer the reliable values of SN parameters.

Below we present the results of a hydrodynamic modeling of SN 2008in event, using the observational data reported by Roy et al. (2011). The results turn out to be of interest in two respects. The derived SN parameters significantly improve earlier estimates of the ejecta mass and the pre-SN radius. Secondly, we uncover an unexpected problem of the inconsistency between the $\mathrm{H} \alpha$ and $\mathrm{H} \beta$ lines in early spectrum, which has interesting implications for the structure of external ejecta.

We begin with the description of the model and the observational data of SN 2008in (Sect. 2). We then turn to the results of the hydrodynamic modeling (Sect. 3.1) and consider the modeling of hydrogen lines in the early spectrum (Sect. 3.2). Section 4 summarizes the results obtained and discusses them in the context of other SNe IIP.

Below we adopt, following Roy et al. (2011), the distance to $\mathrm{SN} 2008$ in $D=13.19 \mathrm{Mpc}$ and the reddening $E(B-V)=$ 0.098 mag. 

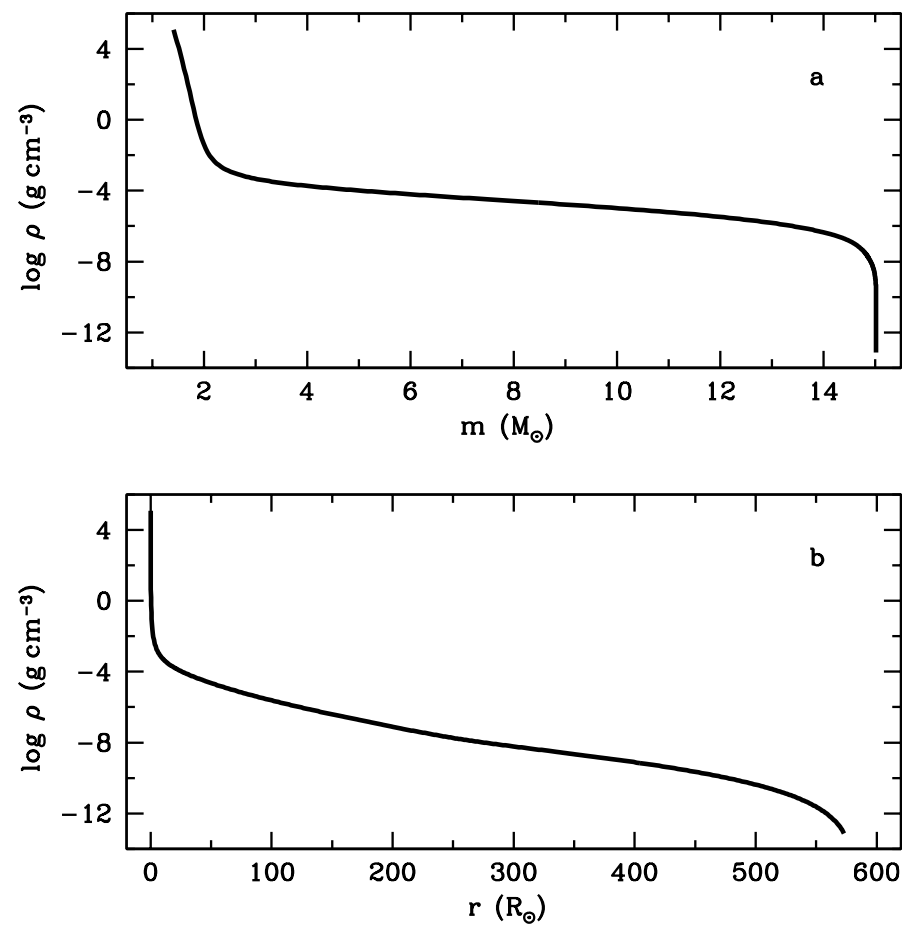

Fig. 1. Density distribution as a function of interior mass (Panel a)) and radius (Panel b)) for the optimal pre-SN model of SN 2008in. The central core of $1.4 M_{\odot}$ is omitted.

\section{Model and observational data}

The modeling of the SN explosion was performed using the spherically-symmetric hydrodynamic code with one-group radiation transfer (Utrobin 2004, 2007), which has been applied previously to other SNe IIP. When applied to the normal type IIP SN 1999em (Utrobin 2007), the code leads to the basic SN parameters similar to those recovered by Baklanov et al. (2005) who employ the hydrodynamics with multi-group radiation transfer. This concordance suggests that the SN IIP modeling is not hampered by the one-group approximation for the radiation transfer. The basic equations and details of the input physics, including calculations of mean opacities, are described in Utrobin (2004). The present version of the code includes additional Compton cooling and heating.

It is general wisdom that a normal SN IIP originates from an explosion of a massive red supergiant (RSG) star (Grassberg et al. 1971; Falk \& Arnett 1977; Eastman et al. 1994). Although SN 2008in is an underluminous SN IIP, its light curve and expansion velocities leave no doubt that this event is caused by the explosion of a RSG star as well. In order to study SN 2008in, we construct, as usual, a non-evolutionary RSG pre-SN model in hydrostatic equilibrium. There are two arguments in favor of non-evolutionary pre-SN models (Utrobin \& Chugai 2008). Firstly, the available evolutionary pre-SN models are not able to describe the shape of the light curve, especially at the end of the plateau. This problem was originally met in the case of SN 1987A (Woosley 1988; Shigeyama \& Nomoto 1990) and was solved by invoking a mixing between the helium core and the hydrogen envelope. Secondly, the multi-dimensional hydrodynamic simulations (Müller et al. 1991; Kifonidis et al. 2003; Hammer et al. 2010) demonstrate that the SN IIP explosion is indeed accompanied by mixing and smoothing of the density and composition gradients between the helium core and the hydrogen envelope. Because the mixing caused by the explosion is

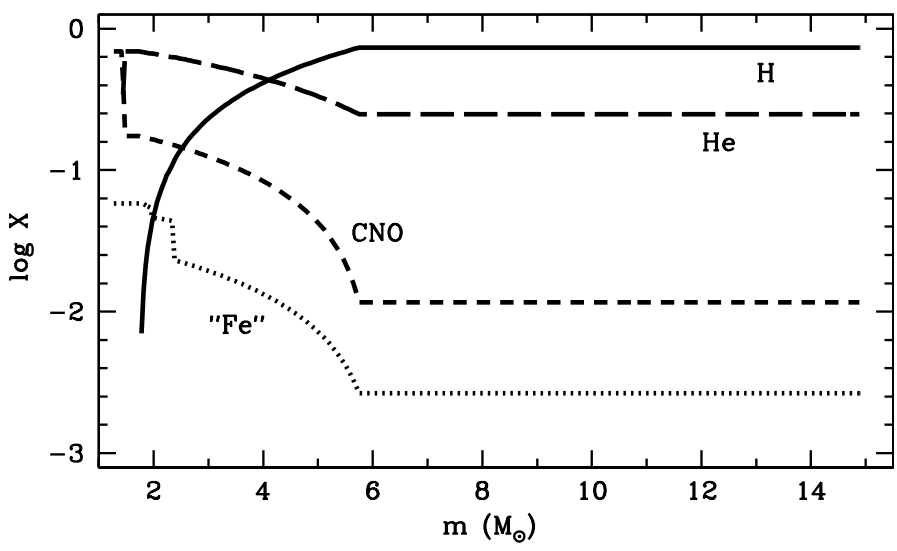

Fig. 2. The mass fraction of hydrogen (solid line), helium (long dashed line), CNO elements (short dashed line), and Fe-peak elements including radioactive ${ }^{56} \mathrm{Ni}$ (dotted line) in the ejecta of the optimal model.

essentially the multi-dimensional effect, we mimic it by a "handmade" non-evolutionary RSG configuration adjusted to fit the observed light curve and the character of the ejecta expansion. The adopted pre-SN density distribution and the chemical composition are shown in Figs. 1 and 2, respectively. We note that the mixing in our pre-SN model presumably reflects a combined effect of mixing during the stellar evolution (cf. Hirschi et al. 2004) and mixing stimulated by the SN explosion.

The SN explosion is initiated by a supersonic piston applied to the bottom of the stellar envelope at the boundary with the $1.4 M_{\odot}$ central core, which is removed from the computational mass domain and assumed to collapse to become a neutron star. The explosion energy we report below is the difference between the piston energy input and the modulus of the total energy of the envelope outside the collapsing core.

In addition to modeling the SN light curves, we analyze the line profiles with the atmosphere model which is based on the time-dependent ionization and excitation kinetics of hydrogen and other elements, the time-dependent kinetics of molecular hydrogen, and the time-dependent energy balance for the gas temperature (Utrobin \& Chugai 2005). The density distribution, chemical composition, radius of the photosphere, and effective temperature are provided by the hydrodynamic model. The obtained time-dependent structure of the atmosphere is then used to model synthetic spectra at selected epochs. The Sobolev local escape approximation is assumed for the line radiation transfer dominated by the line absorption. The line emissivity and the Sobolev optical depth are determined by level populations provided with the time-dependent approach. The Thomson scattering on thermal electrons, Rayleigh scattering on neutral hydrogen, and the relativistic effects are also taken into account. Photons striking the photosphere are assumed to be diffusively reflected back into the atmosphere with the albedo calculated according to Chugai \& Utrobin (2000). The spectra are simulated by means of the Monte Carlo technique.

The hydrodynamic modeling with the one-group radiation transfer is aimed at reproducing a bolometric light curve. For SN 2008in this light curve is recovered using UBVRIJH photometry (Roy et al. 2011) corrected for the reddening and the zero-points reported by Bessell et al. (1998). As usual, we use a black-body spectral fit, which is not a perfect procedure. To estimate errors of the observed bolometric light curve we employ a black-body spectrum modified with a variable ultraviolet reduction factor derived from the SN 1987A spectral data 

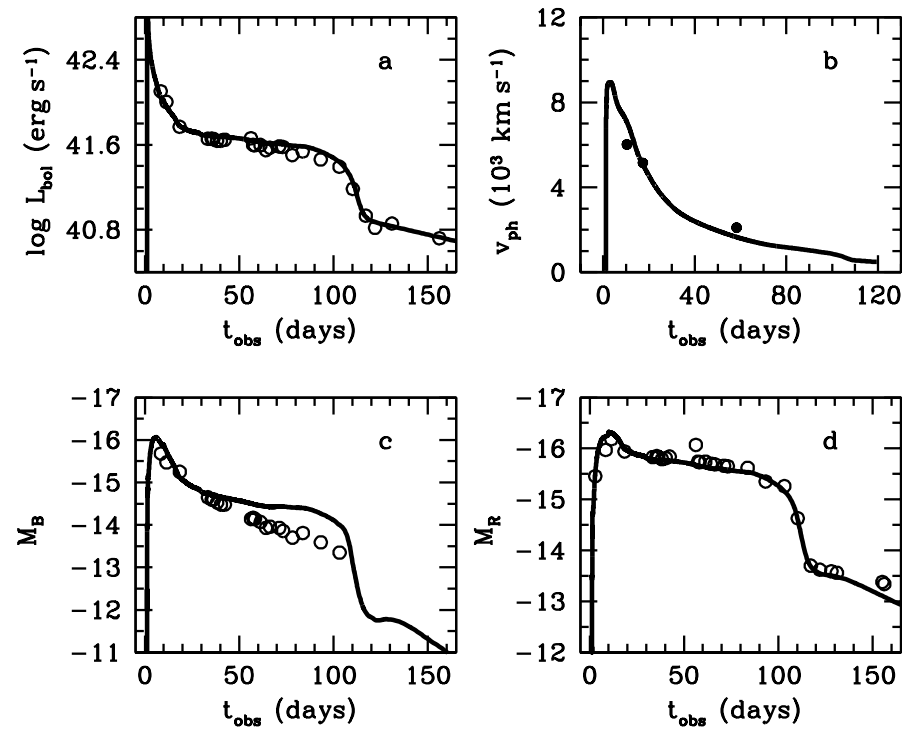

Fig. 3. Optimal hydrodynamic model. Panel a) the bolometric light curve of the optimal model (solid line) overplotted on the bolometric data of SN 2008in (open circles) evaluated from the UBVRIJH magnitudes reported by Roy et al. (2011). Panel b) the calculated photospheric velocity (solid line) is compared to the photospheric velocity estimated from the $\mathrm{H} \alpha, \mathrm{H} \beta, \mathrm{He}$ I $5876 \AA$, and $\mathrm{Na}$ I doublet profiles in the spectra presented by Roy et al. (filled circles). Panels c) and d) the calculated $\mathrm{B}$ and $\mathrm{R}$ light curves (solid line) compared to the observations of SN 2008in (open circles) obtained by Roy et al.

(Phillips et al. 1988; Pun et al. 1995). We find that the black-body fit for SN 2008in results in a slightly higher bolometric luminosity than the modified black-body fit. The difference is less than $5 \%$ for the first 20 days and increases up to $10 \%$ at the end of the plateau. We do not consider this difference to represent an actual error, however, because the SN 1987A spectra show an infrared excess in the $K$ and $L$ bands over the black-body approximation derived from the $B V R I H$ data (Catchpole et al. 1987), which means that the real errors related to the black-body fit are likely to be smaller than our estimates.

The velocity at the photosphere level is another crucial observable constraining the hydrodynamic model. We derive the photospheric velocity via fitting the spectral line profiles by a simple model described in Sect. 3.2. Using the $\mathrm{H} \alpha, \mathrm{H} \beta$, and He I $5876 \AA$ lines on day 11 , the $\mathrm{H} \beta$ line on day 18 , and the $\mathrm{Na}$ I doublet profile on day 59 , we find the photospheric velocity of 6020,5150 , and $2100 \mathrm{~km} \mathrm{~s}^{-1}$ with the uncertainty of $\pm 100 \mathrm{~km} \mathrm{~s}^{-1}$ for these three epochs.

As we will see below, our hydrodynamic model is able to fit not only the bolometric light curve but the $R$-band light curve as well. This is a remarkable result, since the SN detection in the ROTSE-IIIb ${ }^{1}$ images gives the first point of the $R$-band light curve (Roy et al. 2011). Synchronizing the calculated $R$ light curve with the first point observed (Fig. 3d) suggests the explosion date to be JD $=2454822.0$, nearly four days earlier than that accepted by Roy et al. Henceforth we adopt our estimate of the explosion date.

\footnotetext{
The Robotic Optical Transient Search Experiment (ROTSE-III) is a set of four, $45-\mathrm{cm}$, fully robotic optical telescopes.
}

\section{Results}

\subsection{Supernova parameters}

A search for the optimal model is facilitated by the study of parameter variations for the hydrodynamic model of the normal type IIP SN 1999em described earlier (Utrobin 2007). This knowledge combined with a sample of the hydrodynamic models for the well-observed SNe IIP (Utrobin \& Chugai 2011) provide us with efficient recipes to search for the optimal SN parameter set. We note that the radioactive ${ }^{56} \mathrm{Ni}$ mass, which is an essential parameter, can be recovered in a model-independent way from the bolometric luminosity at the radioactive tail. This procedure results in the ${ }^{56} \mathrm{Ni}$ mass of $0.015 M_{\odot}$ in a very good agreement with Roy et al. (2011). Another approach employs a comparison of the SN 2008in luminosity in the $R$ band to that of SN 1987A at the same epoch. This method gives a slightly lower ${ }^{56} \mathrm{Ni}$ mass of $0.012 M_{\odot}$, when adopting the value of $0.076 M_{\odot}$ for SN 1987A (Utrobin 2005). Our experience with SN 2005cs (Utrobin \& Chugai 2008), resembling SN 2008in in the photometric and spectral properties, suggests that the pre-SN radius of SN 2008in should lie between 460 and $740 R_{\odot}$. The preliminary simulations of the light curve and the expansion velocities for SN 2008in show that the appropriate model has the ejecta mass in the range of $12.5-14.5 M_{\odot}$ and the explosion energy in the range of (4-6) $\times 10^{50} \mathrm{erg}$. For the detailed analysis we therefore adopt the helium core mass of $\sim 4 M_{\odot}$, typical for the non-rotating pre-SN model of a $15 M_{\odot}$ main-sequence progenitor (Hirschi et al. 2004). It should be noted that the light curve is not sensitive to the mass of the helium core; instead the light curve depends on a total ejecta mass and a degree of mixing between the helium core and the hydrogen envelope (Utrobin 2007).

The hydrodynamic modeling of SN 2008in for an extended parameter set led us to the optimal choice of the ejecta mass $M_{\text {env }}=13.6 M_{\odot}$, the explosion energy $E=5.05 \times 10^{50} \mathrm{erg}$, the pre-SN radius $R_{0}=570 R_{\odot}$, and the radioactive ${ }^{56} \mathrm{Ni}$ mass $M_{\mathrm{Ni}}=0.015 M_{\odot}$. This is demonstrated by a good fit of the calculated bolometric light curve and the photospheric velocity to those observed (Fig. 3). The inferred ejecta mass and the explosion energy are close to the values found earlier by Roy et al. (2011) employing scaling relations. However, our pre-SN radius is four times larger. The latter suggests that the SN parameters derived from the scaling relations should be cautiously accepted. It should be emphasized that the broad initial luminosity peak (Fig. 3a) directly indicates the explosion of an extended RSG star, not a compact one. While the overall evolution of the velocity at the photosphere level is well reproduced (Fig. 3b), on day 11 the model velocity is $20 \%$ higher than the observed value. This mismatch may be related to the $\mathrm{H} \alpha$ and $\mathrm{H} \beta$ problem we discuss below in Sect. 3.2.

The model density distribution in the freely expanding envelope on day 50 (Fig. 4) is similar to that of SN 2005cs (Utrobin $\&$ Chugai 2008) with the outer density power law $\rho \propto v^{-7.6}$. The power-law index $k=-\partial \ln \rho / \partial \ln v$ depends on the density distribution of pre-SN outer layers, which in turn is constrained by the initial luminosity peak. The rule of thumb states that a more luminous and longer initial luminosity peak requires a shallower density distribution in the outer layers, i.e., a lower $k$ value. In the case of SN 2008in, the $k$ value is determined with an accuracy of about \pm 0.3 . It is worth noting that the modeling of three SNe IIP, namely, SN 2004et (Utrobin \& Chugai 2009), SN 2005cs (Utrobin \& Chugai 2008), and SN 2008in, results in a similar density gradient with $k \approx 7.6$ in the outer layers. 


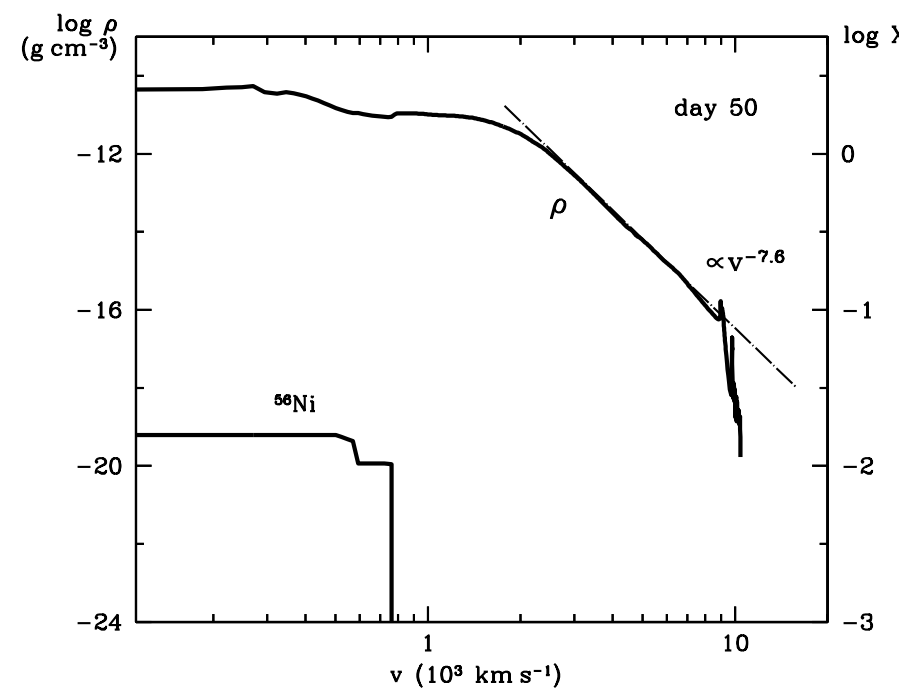

Fig. 4. The density and the ${ }^{56} \mathrm{Ni}$ mass fraction as a function of velocity for the optimal model at $t=50$ days (solid lines). Dash-dotted line is the density distribution fit $\rho \propto v^{-7.6}$.

The principal parameters $\left(M_{\mathrm{env}}, E\right.$, and $\left.R_{0}\right)$ of SN 2008in are similar to those of SN 2005cs (Utrobin \& Chugai 2008; Table 1), although the ejecta mass is somewhat lower and closer to that of the low-luminosity type IIP SN 2003Z (Utrobin et al. 2007). The ${ }^{56} \mathrm{Ni}$ mass in SN 2008in is higher than that of SN 2005cs by a factor of two. Remarkably, the outer velocity of ${ }^{56} \mathrm{Ni}$ material of $770 \mathrm{~km} \mathrm{~s}^{-1}$ also exceeds that of SN $2005 \mathrm{cs}$ by $160 \mathrm{~km} \mathrm{~s}^{-1}$.

Combining the ejecta mass with the mass of the neutron star, we obtain the pre-SN mass of $15 M_{\odot}$. This is the lower limit of a ZAMS progenitor mass because some amount is lost via the stellar wind. Following our previous estimate for SN $2003 Z$ (Utrobin et al. 2007), we adopt the amount of the lost mass to be $0.2<M_{w}<0.8 M_{\odot}$. With this value of the lost mass, the progenitor mass of SN 2008in is then $M=15.5 \pm 0.3 M_{\odot}$.

The question of possible errors of the parameter value is crucial. We estimate the errors by calculating the hydrodynamic models with the parameters varied around the optimal model. Adopting the uncertainties of the observables to be $35 \%$ in the luminosity, $2 \%$ in the velocity, and $1 \%$ in the plateau duration, we come to the errors in the initial radius of $\pm 100 R_{\odot}$, the ejecta mass of $\pm 1.9 M_{\odot}$, the explosion energy of $\pm 3.4 \times 10^{50} \mathrm{erg}$, and the ${ }^{56} \mathrm{Ni}$ mass of $\pm 0.005 M_{\odot}$. We note that the largest error in the explosion energy is related to the large uncertainty of the observed luminosity. The error of the ejecta mass combined with the uncertainty of the mass loss results in the error of the progenitor mass of $\pm 2.2 M_{\odot}$.

\subsection{Hydrogen line problem}

To recover the information on the external rarefied layers imprinted in the $\mathrm{H} \alpha$ wings, we solved the time-dependent atmosphere model of the hydrogen ionization and excitation upon the background of the optimal hydrodynamic model (for details see Utrobin \& Chugai 2005). The results obtained in this way are a little odd (Fig. 5). While the late $\mathrm{H} \alpha$ profile on day 59 is reproduced satisfactorily, the early profile on day 11 is very different from the observed line. The major drawback of the model is a pronounced blueshift, which is also apparent in the $\mathrm{H} \beta$ line on day 11 (see the inset in Fig. 5a). The attempt to vary the hydrodynamics of the outer layers does not remove the problem.
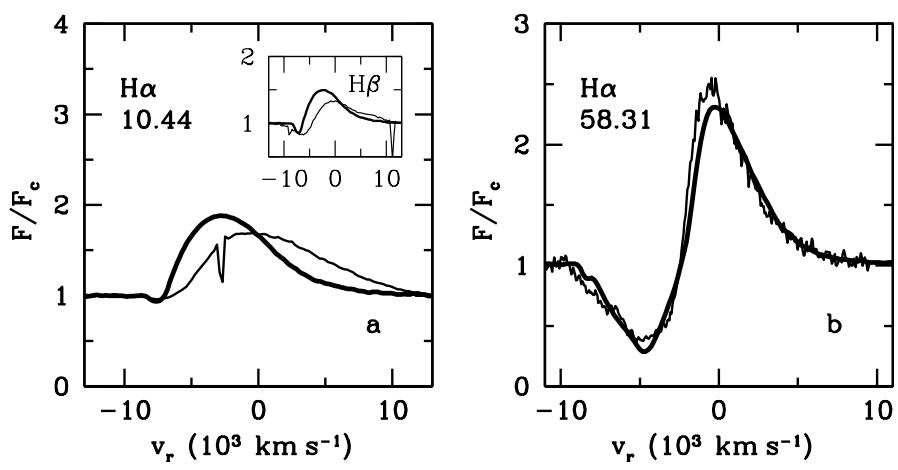

Fig. 5. The $\mathrm{H} \alpha$ profile (thick solid line) on days 11 and 59, calculated with the atmosphere model, is overplotted on the observed profile (thin solid line) (Roy et al. 2011). The inset in Panel a) shows the $\mathrm{H} \beta$ profiles on day 11 .
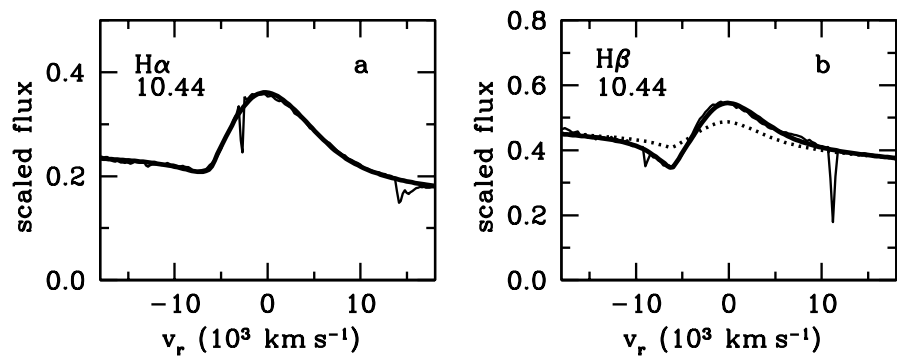

Fig. 6. The best-fit simulations of the $\mathrm{H} \alpha$ and $\mathrm{H} \beta$ lines (thick lines) are overplotted on the corresponding profiles observed on day 11 (thin lines). Unlike the calculations shown in Fig. 5, here we use a simple model with the radial distributions of the Sobolev optical depth and the line emissivity, which are similar for the $\mathrm{H} \alpha$ and $\mathrm{H} \beta$ lines, but scaled arbitrarily to fit both profiles. The dotted line represents the $\mathrm{H} \beta$ line for the theoretical ratio $R_{\tau}=7.25$.

The first thought is that the uncovered mismatch could be an outcome of a large velocity at the photosphere level in the hydrodynamic model; this could be responsible for the strong occultation effect. Indeed, we found earlier that on day 11 the empirical velocity of $6020 \mathrm{~km} \mathrm{~s}^{-1}$ is lower than the model value of $7130 \mathrm{~km} \mathrm{~s}^{-1}$ (Fig. 3b). However, we show below that this is not the principal reason. Here we notice that a small dip in the blue absorption wing of the model $\mathrm{H} \alpha$ profile on day 59 at the radial velocity of $-8200 \mathrm{~km} \mathrm{~s}^{-1}$ (Fig. 5b) is the trace of the boundary shell with a mass of $\sim 10^{-3} M_{\odot}$, which forms during the shock breakout (Fig. 4). A similar dip is observed in some SNe IIP (Chugai et al. 2007), but is absent in SN 2008in.

A question arises whether the difference between the model profile and the observed line on day 11 is an outcome of the inadequate distribution of the ejecta density, the ionization, and the excitation in the outer layers, or the result of some other factors. To explore this issue, we use a parametrized model of the line formation, which admits a variation of the distributions of the Sobolev optical depth and the line emissivity in a wide range. The ratio of the Sobolev optical depth of the $\mathrm{H} \alpha$ and $\mathrm{H} \beta$ lines is a constant determined by atomic data $R_{\tau}=\tau_{23} / \tau_{24}=7.25$. This ratio can be slightly modified by the stimulated emission; this effect is included in the numerical computations. A surprising result of these simulations is that both $\mathrm{H} \alpha$ and $\mathrm{H} \beta$ lines cannot be described simultaneously in the framework of a spherical model. The profiles can be reproduced if and only if we abandon the theoretical ratio $R_{\tau}=7.25$ : the best fit of the $\mathrm{H} \alpha$ and $\mathrm{H} \beta$ profiles on day 11 (Fig. 6) is attained for the ratio $R_{\tau}=2.5$, three times lower. To put it simply, the $\mathrm{H} \alpha$ absorption component 

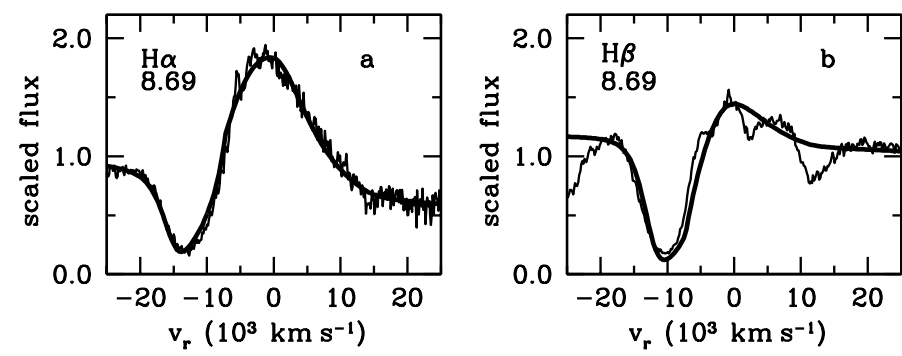

Fig. 7. The $\mathrm{H} \alpha$ and $\mathrm{H} \beta$ lines observed in SN 1987A on day 9 (thin lines) (Phillips et al. 1988) and the calculated profiles (thick lines) in the simple model with the theoretical ratio $R_{\tau}=7.25$.

Table 1. Hydrodynamic models of type IIP supernovae.

\begin{tabular}{lcccccc}
\hline \hline$S N$ & $\begin{array}{c}R_{0} \\
\left(R_{\odot}\right)\end{array}$ & $\begin{array}{c}M_{\text {env }} \\
\left(M_{\odot}\right)\end{array}$ & $\begin{array}{c}E \\
\left(10^{51} \mathrm{erg}\right)\end{array}$ & $\begin{array}{c}M_{\mathrm{Ni}} \\
\left(10^{-2} M_{\odot}\right)\end{array}$ & $\begin{array}{c}v_{\mathrm{Ni}}^{\max } \\
\left(\mathrm{km} \mathrm{s}^{-1}\right)\end{array}$ \\
\hline $1987 \mathrm{~A}$ & 35 & 18 & 1.5 & 7.65 & 3000 & 600 \\
$1999 \mathrm{em}$ & 500 & 19 & 1.3 & 3.6 & 660 & 700 \\
$2000 \mathrm{cb}$ & 35 & 22.3 & 4.4 & 8.3 & 8400 & 440 \\
$2003 \mathrm{Z}$ & 230 & 14 & 0.245 & 0.63 & 535 & 360 \\
$2004 \mathrm{et}$ & 1500 & 22.9 & 2.3 & 6.8 & 1000 & 300 \\
$2005 \mathrm{cs}$ & 600 & 15.9 & 0.41 & 0.82 & 610 & 300 \\
$2008 \mathrm{in}$ & 570 & 13.6 & 0.505 & 1.5 & 770 & 490 \\
$2009 \mathrm{kf}$ & 2000 & 28.1 & 21.5 & 40.0 & 7700 & 410 \\
\hline
\end{tabular}

is significantly weaker than expected from the strength of the $\mathrm{H} \beta$ absorption. To emphasize the apparent oddity of this phenomenon, we show the $\mathrm{H} \alpha$ and $\mathrm{H} \beta$ lines in the spectra of SN 1987A on day 9 (Phillips et al. 1988) and their reasonable fit for the theoretical ratio of the Sobolev optical depths (Fig. 7).

We have no ready explanation for the disparity found between the $\mathrm{H} \alpha$ and $\mathrm{H} \beta$ lines. Two suggestions are conceivable and both are related to deviations from the spherical symmetry. For instance, the outer layers are admittedly clumpy. In that case the effective optical depth of a line is determined not only by the atomic cross-sections, but also by the clumpiness parameters. For optically thick clumps, a situation is plausible when the strengths of the $\mathrm{H} \alpha$ and $\mathrm{H} \beta$ absorptions become comparable. Thus, it imitates the case that we find in the early spectrum of SN 2008in. Another possibility is that a large-scale emission asymmetry in the near hemisphere could originate from either the overall ejecta asymmetry or the asymmetric ${ }^{56} \mathrm{Ni}$ ejecta, both being presumably related to the explosion asymmetry. This asymmetry might produce the found disparity if the contribution of the emission asymmetry is significant in the $\mathrm{H} \alpha$ line and rather weak in the $\mathrm{H} \beta$ line. It is quite plausible given the smaller optical depth in the $\mathrm{H} \beta$ line and the conversion of the $\mathrm{H} \beta$ photons into the $\mathrm{P} \alpha$ and $\mathrm{H} \alpha$ quanta. The $\mathrm{H} \alpha$ emission asymmetry can fill in the absorption component, thus resulting in the week $\mathrm{H} \alpha$ absorption. We kept ourselves to these general remarks and plan to study the $\mathrm{H} \alpha$ vs. $\mathrm{H} \beta$ disparity in detail elsewhere.

\section{Discussion and conclusions}

The primary goal of the paper was to recover the parameters of the subluminous type IIP SN 2008in from the observational data of Roy et al. (2011) using hydrodynamic simulations. We find the ejecta mass $M_{\mathrm{env}}=13.6 \pm 1.9 M_{\odot}$, the explosion energy $E=(5.05 \pm 3.4) \times 10^{50} \mathrm{erg}$, and the pre-SN radius $R_{0}=570 \pm 100 R_{\odot}$. The ${ }^{56} \mathrm{Ni}$ mass estimated from the radioactive tail is $M_{\mathrm{Ni}}=0.015 \pm 0.005 M_{\odot}$. The earlier estimates of $E$

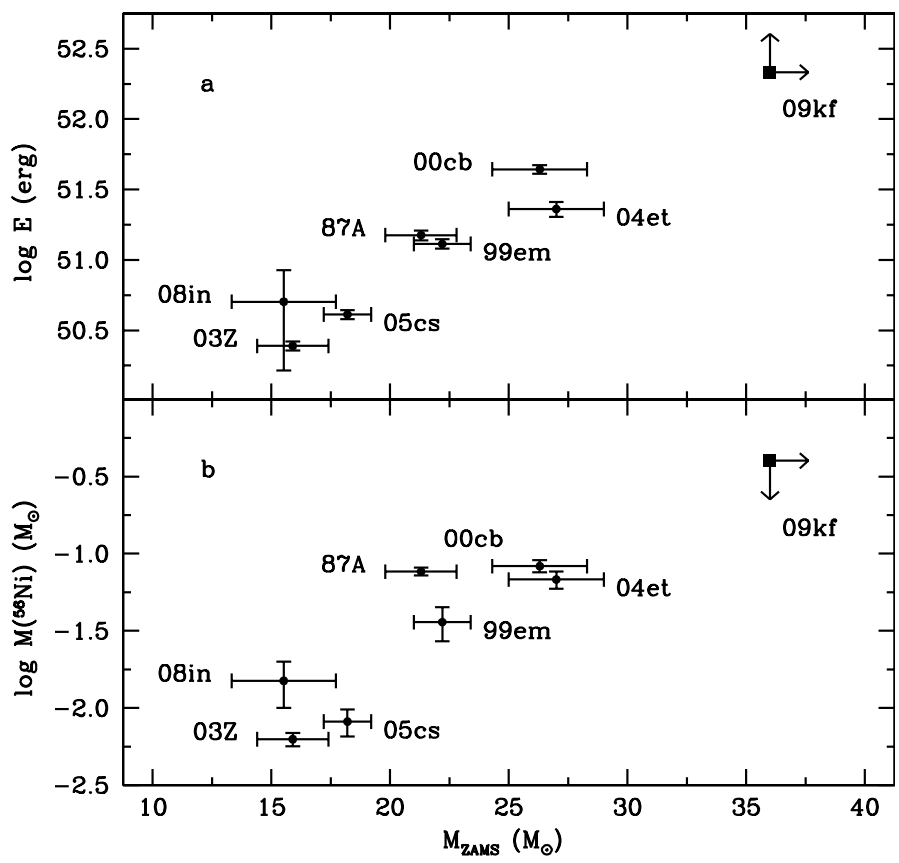

Fig. 8. Explosion energy (Panel a)) and ${ }^{56} \mathrm{Ni}$ mass (Panel b)) vs. hydrodynamic progenitor mass for SN 2008in and seven other core-collapse SNe (Utrobin \& Chugai 2011).

and $M_{\mathrm{Ni}}$ reported by Roy et al. (2011) are in reasonable agreement with our results, although our value of the ejecta mass is $3 M_{\odot}$ lower than that of Roy et al. More importantly, we do not confirm the earlier conclusion that the pre-SN was a relatively compact: our pre-SN radius of $570 R_{\odot}$ suggests that the pre-SN was a normal RSG star. Taking the mass of the neutron star and the mass loss into account, we estimate the progenitor mass to be $M_{\text {ZAMS }}=15.5 \pm 2.2 M_{\odot}$.

The parameters of SN 2008in are similar to those of another underluminous type IIP SN 2005cs (Table 1). The only significant difference is that the ${ }^{56} \mathrm{Ni}$ mass in SN 2008in is a factor of two higher than that of SN 2005cs. It is a somewhat puzzling result given the higher or at least comparable ejecta mass of SN 2005cs. This indicates that the explosion conditions, imprinted in the amount of synthesized ${ }^{56} \mathrm{Ni}$, are not a unique function of the progenitor mass of SNe IIP.

It is instructive to place SN 2008in on the diagrams of the explosion energy vs. the progenitor mass and the ${ }^{56} \mathrm{Ni}$ mass vs. the progenitor mass (Fig. 8) together with all the rest of SNe IIP studied hydrodynamically (Utrobin \& Chugai 2011). We note that the error in the explosion energy for SN 2008in, which is larger than for other SNe IIP, is related to the larger uncertainty in the distance combined with the reddening error. The supernova 2008in falls into a broad strip occupied by SNe IIP, thus confirming an assumption that the explosion energy and the ${ }^{56} \mathrm{Ni}$ mass correlate with the progenitor mass $M_{\text {ZAMS }}$. The available sample of the hydrodynamically studied SNe IIP is relatively scarce, and a larger number of such events is needed to confirm and to highlight these correlations, which are of great importance for constraining the explosion mechanism.

We face an unexpected problem: the time-dependent model of the hydrogen ionization and the excitation, computed on the hydrodynamics background, fails to reproduce the $\mathrm{H} \alpha$ and $\mathrm{H} \beta$ lines in the SN 2008in spectrum on day 11. At first glance, this indicates that the radial structure of the outermost layers in SN 2008in differs substantially from the model hydrodynamic flow. However, it turns out that the problem has deeper roots. 
Detailed study shows that there is no way to reproduce the $\mathrm{H} \alpha$ and $\mathrm{H} \beta$ lines simultaneously in the framework of a spherically symmetric model. We consider this as evidence that at least the outer ejecta $\left(v \geq 7000 \mathrm{~km} \mathrm{~s}^{-1}\right)$ are not spherical: a clumpiness and/or global asymmetry essentially affects the hydrogen line formation in the high-velocity layers.

The global asphericity of the $\mathrm{H} \alpha$-emitting ejecta could be realized as a non-spherical pattern of the hydrogen ionization and the excitation produced by the asymmetric ${ }^{56} \mathrm{Ni}$ ejecta. The strong asymmetry of ${ }^{56} \mathrm{Ni}$ ejecta was observed in the type IIP SN 2004dj (Chugai et al. 2005) and to a lesser extent in SN 1987A (Haas et al. 1990). Although little can be said in detail on how this asymmetry is produced, it could be related to the explosion asymmetry.

The clumpiness of the ejecta is the well-known phenomenon among core-collapse SNe. Particularly, the oxygen ejecta show clearly a clumpy structure in [OI] 6300 and $6363 \AA$ lines of the type IIP SN 1987A (Stathakis et al. 1991) and the type Ib/c SN 1985F (Filippenko 1991). Less apparent is the situation with the clumpiness of the outer ejecta. At the late $(t \geq 1 \mathrm{yr})$ epoch, the spectra of the type IIb SN 1993J show a clumpy structure of the $\mathrm{H} \alpha$-emitting shell (Filippenko et al. 1994), although it is not certain whether this clumpiness was produced during the SN outburst or the circumstellar interaction. When studying the Cas $\mathrm{A}^{2}$ morphology, Fesen (2001) detects the high-velocity $\left(\approx 10^{4} \mathrm{~km} \mathrm{~s}^{-1}\right)$ nitrogen knots containing hydrogen. This finding suggests that the external ejecta of the Cas A parent $\mathrm{SN}$ are clumpy. We therefore conclude that the global asymmetry caused by the ${ }^{56} \mathrm{Ni}$ ejecta and/or the clumpiness of the outer layers could be considered a possible explanation of the $\mathrm{H} \alpha$ and $\mathrm{H} \beta$ disparity. A detailed study is needed to resolve the issue.

To our knowledge, the $\mathrm{H} \alpha$ and $\mathrm{H} \beta$ disparity revealed for SN 2008in has not been ever mentioned for any SN IIP and at the moment we cannot say whether this problem is characteristic of other SNe IIP as well. It is worth noting that the computed $\mathrm{H} \alpha$ emission shows a strong blueshift compared to that observed in the early spectra of SN 2005cs (Dessart et al. 2008). We are not sure, however, whether this disparity for SN $2005 \mathrm{cs}$ is fatal, or if it could be eliminated by the appropriate tuning of a spherical model. On the other hand, we know that the $\mathrm{H} \alpha$ and $\mathrm{H} \beta$ disparity is absent in SN 1987A. This gives us a clue that the dissimilarity of the behavior of the $\mathrm{H} \alpha$ and $\mathrm{H} \beta$ lines in SN 2008in and SN 1987A is presumably related to a different structure of their pre-SNe. Indeed, the shock breakout is likely to be accompanied by the fragmentation of the low-mass boundary shell. One expects a more massive fragmented shell in the case of an exploding RSG star $\left(\sim 10^{-3} M_{\odot}\right.$; Sect. 3.2) than for a blue supergiant pre-SN $\left(\sim 10^{-6} M_{\odot}\right.$; Imshennik \& Nadezhin 1989). If the $\mathrm{H} \alpha$ and $\mathrm{H} \beta$ disparity is related to the clumpy structure of the outer ejecta, the difference in the mass of the fragmented shell might thus be responsible for the difference of the $\mathrm{H} \alpha$ and $\mathrm{H} \beta$ behavior in these SNe IIP.
The ejecta mass of SN 2008in is the smallest among those derived by the hydrodynamic modeling. Even with the conservative estimate of the progenitor mass of $15.5 \pm 2.2 M_{\odot}$, we face a challenging problem: why does not the sample of the wellobserved SNe IIP include the events with masses $\leq 15 M_{\odot}$ ? This problem has already been discussed by Utrobin \& Chugai (2008) and is summarized as follows: either the observed sample is biased towards the luminous, high-mass SNe IIP or the hydrodynamic masses are overestimated for an unknown reason. At this stage both explanations seem plausible which leaves open the question of the progenitor mass range recovered for SNe IIP by hydrodynamic modeling.

Acknowledgements. We thank Rupak Roy for kindly sending us spectra of SN 2008in. V.P.U. is grateful to Wolfgang Hillebrandt for the possibility of working at the MPA. We also thank the anonymous referee for critical comments which helped improve the manuscript.

\section{References}

Baklanov, P. V., Blinnikov, S. I., \& Pavlyuk, N. N. 2005, Astron. Lett., 31, 429 Bessell, M. S., Castelli, F., \& Plez, B. 1998, A\&A, 333, 231

Catchpole, R. M., Menzies, J. W., Monk, A. S., et al. 1987, MNRAS, 229, 15P Chugai, N. N., \& Utrobin, V. P. 2000, A\&A, 354, 557

Chugai, N. N., Fabrika, S. N., Sholukhova, O. N., et al. 2005, Astron. Lett., 31, 792

Chugai, N. N., Chevalier, R. A., \& Utrobin, V. P. 2007, ApJ, 662, 1136

Dessart, L., Blondin, S., Brown, P. J., et al. 2008, ApJ, 675, 644

Eastman, R. G., Woosley, S. E., Weaver, T. A., \& Pinto, P. A. 1994, ApJ, 430, 300

Falk, S. W., \& Arnett, W. D. 1977, A\&AS, 33, 515

Fesen, R. A. 2001, ApJS, 133, 161

Filippenko, A. V. 1991, in Supernovae, ed. S. E. Woosley, 467

Filippenko, A. V., Matheson, T., \& Barth, A. J. 1994, AJ, 108, 2220

Grassberg, E. K., Imshennik, V. S., \& Nadyozhin, D. K. 1971, Ap\&SS, 10, 28

Haas, M. R., Erickson, E. F., Lord, S. D., et al. 1990, ApJ, 360, 257

Hammer, N. J., Janka, H.-T., \& Müller, E. 2010, ApJ, 714, 1371

Heger, A., Fryer, C. L., Woosley, S. E., Langer, N., \& Hartmann, D. H. 2003, ApJ, 591, 288

Hirschi, R., Meynet, G., \& Maeder, A. 2004, A\&A, 425, 649

Imshennik, V. S., \& Nadezhin, D. K. 1989, Astrophys. Space Phys. Rev., 8, 1

Kifonidis, K., Plewa, T., Janka, H.-T., \& Müller, E. 2003, A\&A, 408, 621

Krause, O., Birkmann, S. M., Usuda, T., et al. 2008, Science, 320, 1195

Müller, E., Fryxell, B., \& Arnett, D. 1991, A\&A, 251, 505

Phillips, M. M., Heathcote, S. R., Hamuy, M., \& Navarrete, M. 1988, AJ, 95, 1087

Pun, C. S. J., Kirshner, R. P., Sonneborn, G., et al. 1995, ApJS, 99, 223

Roy, R., Kumar, B., Benetti, S., et al. 2011, ApJ, 736, 76

Shigeyama, T., \& Nomoto, K. 1990, ApJ, 360, 242

Smith, N., Li, W., Filippenko, A. V., \& Chornock, R. 2011, MNRAS, 412, 1522

Stathakis, R. A., Dopita, M. A., Cannon, R. D., \& Sadler, E. M. 1991, in Supernovae, ed. S. E. Woosley, 95

Utrobin, V. P. 2004, Astron. Lett., 30, 293

Utrobin, V. P. 2005, Astron. Lett., 31, 806

Utrobin, V. P. 2007, A\&A, 461, 233

Utrobin, V. P., \& Chugai, N. N. 2005, A\&A, 441, 271

Utrobin, V. P., \& Chugai, N. N. 2008, A\&A, 491, 507

Utrobin, V. P., \& Chugai, N. N. 2009, A\&A, 506, 829

Utrobin, V. P., \& Chugai, N. N. 2011, A\&A, 532, A100

Utrobin, V. P., Chugai, N. N., \& Pastorello, A. 2007, A\&A, 475, 973

Woosley, S. E. 1988, ApJ, 330, 218

\footnotetext{
2 Cas $\mathrm{A}$ is the SN remnant presumably produced by the explosion of the type IIb SN (Krause et al. 2008).
} 